\title{
Исследования структурных характеристик мозга в психогенетике
}

\author{
Илья М. Захаров*, Сергей Б. Малых \\ Федеральное государственное бюджетное научное учреждение «Психологический институт Российской \\ академии образования», г. Москва, Российская Федерация \\ *E-mail: iliazaharov@gmail.com \\ ORCID ID: https://orcid.org/0000-0001-7207-9641, https://orcid.org/0000-0002-3786-7447
}

\begin{abstract}
Аннотация
Ввеление. В статье представлен обзор современных исслеАований природы инАивиАуальных различий структурных характеристик мозга. В рамках концепции эндофренотипов (промежуточного звена межАу геном и комп^ексным феенотипическим признаком) рассматривается роль инАивиАуальных различий в структурных характеристиках мозга в фрормировании инАивиАуальных различий в психологических признаках.

Теоретическое обоснование. В настоящей работе анализируется роль генетических и среАовых фракторов в фрормировании инАивиАуальных различий в структурных характеристиках мозга, измеренных С помощью мето Аов магнитно-резонансной томографрии и Аифрфрузно-тензорной визуализации. В обзор вк^ючены результаты близнецовых исслеАований, исслеАований генов-канАиАатов, а также полногеномных исслеАований ассоциаций.

Результаты и их обсужАение. В целом генетически инорормативные исслеАования структурных характеристик мозга свидетельствуют о том, что Аля небольшого ряла структур (например, проводящие пути кортикоспинального тракта или объем боковых желудочков) наблюАаются умеренные показатели наследуемости (от 20 Ао 50\%), тогАа как наслеАуемость большинства структурных характеристик - более $50 \%$. Показано, что вкАаА генетических фракторов в инАивиАуальные различия структурных характеристик мозга изменяется в холе онтогенеза. На основании применения методов многомерного анализа выявлен общий генетический вкАаА в инАивиАуальные различия в структурных характеристиках мозга и повеАенческих оренотипов. В обзоре представлены результаты новых типов молекулярно-генетических исследований, в первую очередь с применением метола полногеномного анализа ассоциаций, в котором рассматриваются сотни тысяч АНК-маркеров оАновременно. ОбсужАаются также исследования таких генетических фракторов, как вариация числа копий генов и всегеномные (whole-genome) исследования. В обзоре показано, что в связи с наметившимся

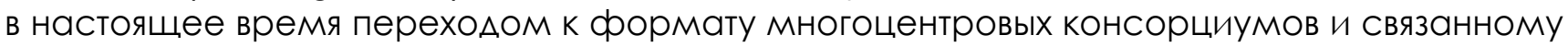
с этим росту исследуемых выборок, у современных исследователей открывается новое поле возможностей Аля изучения вкАаАа генетических фракторов в инАивиАуальные различия в структурных характеристиках мозга.
\end{abstract}

\section{КАючевые слова}

психогенетика, близнецовый метол, GWAS-анализ, гены-кандиАаты, наслеАуемость, томографиические метолы, объем мозга, эндофренотип, интел^ект, шизофррения 
ЗАХАРОВ И. М., МАЛЫХ С. Б.

ИССЛЕДОВАНИЯ СТРУКТУРНЫХ ХАРАКТЕРИСТИК МОЗГА В ПСИХОГЕНЕТИКЕ

РосСИЙскИй психологИЧЕСКИй жУРнАл, 2020, Т. 17, № 2, 17-35. doi: 10.21702/rpj.2020.2.2

МЕДИЦИНСКАЯ ПСИХОЛОГИЯ

\section{Основные положения}

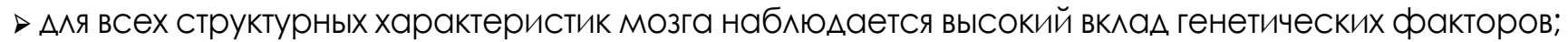
> структурные характеристики мозга на генетическом уровне связаны с рялом повеАенческих характеристик (интелмект, шизоорения, биполярное расстройство и Ар.);

> перехол от близнецовых ИсслеАований к масштабным исслеАованиям на базе межАународных кол^абораций позволяет выявить новые генетические механизмы, ^ежащие в основе инАивиАуальных различий в структурных характеристиках мозга.

\section{Для цитирования}

Захаров, И. М. и Малых, С. Б. (2020). Исследования структурных характеристик мозга в психогенетике. Российский психологический журнал, 17(2), 17-35. doi: 10.21702/rpj.2020.2.2

Дата получения рукописи: 20.05.2020

Дата окончания рецензирования: 17.06 .2020 Дата принятия к публикации: 20.06.2020

\section{Введение}

Один из важнейших источников информации о природе индивидуальных различий - это генетически информативные исследования. Несмотря на принципиальную важность, высокая стоимость и трудоемкость исследований такого типа в течение долгого времени ограничивала их количество. Развитие методов анализа данных и новых технологий исследований деятельности мозга, а также формирование масштабных исследовательских коллабораций, объединяющих большие группы исследователей из разных лабораторий и стран, приводят к всё большему росту интереса к подобным исследованиям.

В 1972 г. И. Готтесманом (Gottesman \& Shields, 1972) была предложена концепция эндофенотипов. В классической генетике фенотип - это любая наблюдаемая или измеряемая изменчивая характеристика (например, рост, вес, цвет глаз, личностные особенности, способности к обучению). Под эндофенотипом понимаются такие показатели, индивидуальные различия которых формируются под влиянием генетических факторов и связаны с психологическими признаками в норме или при психических нарушениях. Эндофенотипы рассматриваются как промежуточное звено между геном и поведением человека, т. е. это звено в цепочке «ген эндофенотип - психологический признак». В качестве эндофенотипов того или иного поведенческого признака может выступать широкий круг различных характеристик - от особенностей морфологии мозга до электрофизиологических феноменов (Малых, Егорова и Мешкова, 2008).

В качестве эндофенотипов психологических характеристик в генетически информативных исследованиях (т.е. в тех исследованиях, в которых учитывается влияние генетических факторов на изучаемый признак) структурных характеристик мозга используется целый ряд показателей, которые регистрируются при использовании различных методов нейровизуализации, таких как магнитно-резонансная томография (МРТ), диффузная тензорная томография и др. Самым популярным на сегодняшний день методом исследования этиологии индивидуальных особенностей морфологии мозга человека является метод МРТ, позволяющий получить трехмерные изображения внутренних структур на основании различий в свойствах ядерного резонанса в разных тканях организма. Другим методом анализа морфологии мозга является метод диффузно-тензорной визуализации (Glahn et al., 2012; Brun et al., 2009; Kochunov et al., 2010). Этот 
ЗАХАРОВ И. М., МАЛЫХ С. Б.

ИССЛЕДОВАНИЯ СТРУКТУРНЫХ ХАРАКТЕРИСТИК МОЗГА В ПСИХОГЕНЕТИКЕ

РосСИЙСКИЙ пСИХОЛОГИЧЕСКИЙ ЖУРнАл, 2020, Т. 17, № 2, 17-35. doi: 10.21702/rpj.2020.2.2

МЕДИЦИНСКАЯ ПСИХОЛОГИЯ

метод позволяет фиксировать пучки нервных волокон, соединяющие различные зоны мозга, а также связи между аксонами белого вещества головного мозга. Метод диффузно-тензорной визуализации (ДТВ) был предложен для оценки анизотропии (направления) в диффузии молекул воды в ткани головного мозга (Chiang et al., 2009).

Несмотря на то, что в последние годы роль структурных характеристик мозга как эндофенотипов психологических признаков подвергалась сомнению (lacono, Malone, \& Vrieze, 2017), совокупный анализ снимков структурной МРТ 21199 человек показал, что особенности строения головного мозга являются значимыми предикторами ряда психических заболеваний, в первую очередь шизофрении, биполярного расстройства и синдрома дефицита внимания и гиперактивности (Thompson et al., 2020). Кроме того, целый ряд исследований показал, что структурные особенности головного мозга связаны с индивидуальными различиями таких психологических фенотипов, как уровень интеллекта (Hilger, Ekman, Fiebach, \& Basten, 2017), особенности эмоциональной сферы (Marstaller, Burianová, \& Reutens, 2016; Adrián-Ventura, Costumero, Parcet, \& Ávila, 2019) и даже демографические характеристики (Llera, Wolfers, Mulders, \& Beckmann, 2019).

В данном обзоре анализируются генетически информативные исследования природы индивидуальных особенностей структурных характеристик мозга, связанных с различными психологическими фенотипами.

\section{Теоретическое обоснование \\ Томографические исследования структурных характеристик мозга с применением близнецового метода}

В психогенетике для оценки относительного вклада генетических и средовых факторов в индивидуальные различия нейрофизиологических признаков используют методы исследования членов семей с различной степенью генетического сходства (Малых, Ковас и Гайсина, 2016). Одним из наиболее информативных методов является сравнение данных, полученных с помощью методов нейровизуализации на монозиготных (M3) и дизиготных (Д3) близнецах. В основе метода лежит различие в генетическом сходстве между МЗ близнецами, вырастающими из одной оплодотворенной яйцеклетки (их сходство составляет $100 \%$ ), и ДЗ близнецами, которые развиваются из двух разных яйцеклеток, оплодотворенных в одно и то же время (у них среднее количество разделенной ДНК равняется $50 \%$ ). Сравнение внутрипарных М3 и Д3 корреляций позволяет оценить роль наследственных и средовых факторов в индивидуальных различиях изучаемого признака. Если генетические факторы вносят вклад в индивидуальные различия в определенном признаке, то корреляция между МЗ близнецами должна быть выше по этому признаку, чем между Д3 близнецами. Для описания вклада генетических факторов в вариабельность признака часто используется термин «наследуемость». Близнецовый метод используется также для оценки этиологии связей между разными признаками на основе применения многомерных моделей, позволяющих оценить вклад генетических и средовых факторов в ковариацию нескольких фенотипических переменных (Малых и др., 2008).

Высокий вклад генетических факторов в индивидуальные различия в структурных характеристиках мозга был показан уже в одном из первых исследований у здоровых М3 и ДЗ близнецов на примере объема латеральных желудочков (Basser, Mattiello, \& Lebihan, 1994). В последующих работах высокие показатели наследуемости были выявлены для таких общих мозговых характеристик, как внутричерепной объем $\left(h^{2}>81 \%\right)$ и общий объем 
ЗАХАРОВ И. М., МАЛЫХ С. Б.

ИССЛЕДОВАНИЯ СТРУКТУРНЫХ ХАРАКТЕРИСТИК МОЗГА В ПСИХОГЕНЕТИКЕ

РосСИЙскИй психологИЧЕСКИй жУРнАл, 2020, Т. 17, № 2, 17-35. doi: 10.21702/rpj.2020.2.2

МЕДИЦИНСКАЯ ПСИХОЛОГИЯ

мозга (66-97 \%) (Baaré et al., 2001; Bartley, Jones, \& Weinberger, 1997; Wright, Sham, Murray, Weinberger, \& Bullmore, 2002; Carmelli et al., 1998; Pfefferbaum, Sullivan, Swan, \& Carmelli, 2000). Существенная роль генетических факторов (вклад более 82 \%) была также показана в первом семейном, включающем как близнецов, так и сиблингов, исследовании индивидуальных различий в общем объеме белого и серого вещества мозга (Reveley, Reveley, Chitkara, \& Clifford, 1984). В других работах были показано, что в вариабельности объема каждого из полушарий 65 \% обусловлено генетическими факторами (Geschwind, Miller, DeCarli, \& Carmelli, 2002; Малых и др., 2016), оценка наследуемости объема мозжечка составила 88 \% (Posthuma et al., 2000), мозолистого тела - 79-94 \% (Pfefferbaum et al., 2000; Scamvougeras, Kigar, Jones, Weinberger, \& Witelson, 2003).

Высокий уровень наследуемости (до 83 \%) также показан для плотности серого вещества в областях Брока и Вернике, лобной коре, извилине Гешля, левой затылочной и левой теменной поясных областях, средней височной коре и миндалине (Cannon et al., 2006; Hulshoff Pol et al., 2006; Thompson et al., 2001). В целом ряде работ была также зафиксирована значительная роль генетических факторов в индивидуальных особенностях толщины коры, особенно во фронтальной и париетальной областях (Joshi et al., 2011; Lenroot et al., 2009; Rimol et al., 2010; Yoon, Fahim, Perusse, \& Evans, 2010). Наиболее высокие показатели генетического вклада (до 93 \%) наблюдаются для индивидуальных различий в плотности белого вещества кортикоспинального тракта, мозолистого тела, верхнего продольного и окципито-фронтального пучков (Thompson et al., 2001; Peper, Zwiers, Boomsma, Kahn, \& Hulshoff Pol, 2009).

Значительное влияние генетических факторов в дисперсии структурных характеристик мозга было также показано на детской выборке (Wallace et al., 2006) и в исследованиях лиц пожилого возраста (69-80 лет) (Carmelli, Swan, DeCarli, \& Reed, 2002). Учитывая, что в пожилом возрасте близнецы разделяют меньшее количество общих средовых факторов (например, проживают раздельно), полученные данные можно интерпретировать в контексте устойчивости генетического контроля объема исследованных мозговых структур.

Кроме МРТ-исследований, важный вклад генетических факторов был показан также в исследованиях с применением метода диффузно-тензорной томографии (ДТТ). Так, на основе использования ДТТ в исследовании с привлечением близнецов и других сиблингов были получены данные о заметной роли наследуемости в изменчивости асимметрии нижнего лобно-теменного пучка (33\%), переднего таламического расхождения (37\%) и крючковидного пучка (20\%). При этом был также показан вклад общих средовых факторов: 10 для малых щипцов и 15\% для кортикоспинального тракта (Jahanshad et al., 2010). Близнецовые исследования с применением диффузно-тензорной томографии также показали значительные показатели наследуемости мозолистого тела (Brun et al., 2009; Pfefferbaum et al., 2000), окципитальных долей коры (Brun et al., 2009), а также латеральной орбитофронтальной извилины, мозжечка, ряда подкорковых структур, ствола головного мозга и крючка головного мозга, правого темпорального белого вещества и верхней лобной извилины (Yoon, Perusse, Lee, \& Evans, 2011).

Проведенный в 2012 г. метаанализ 62 генетически информативных исследований характеристик морфологии структур мозга показал, что в целом генетические факторы вносят значительный вклад в индивидуальные различия в таких общих параметрах, как внутричерепной объем, общий объем мозга, объем коры больших полушарий, общий и локальные объемы серого и белого вещества и др. (Blokland, de Zubicaray, McMahon, \& Wright, 2012). При этом, согласно результатам метаанализа, также наблюдается значительная вариативность 
ЗАХАРОВ И. М., МАЛЫХ С. Б.

ИССЛЕДОВАНИЯ СТРУКТУРНЫХ ХАРАКТЕРИСТИК МОЗГА В ПСИХОГЕНЕТИКЕ

РосСИЙСКИЙ пСИХОЛОГИЧЕСКИЙ ЖУРнАл, 2020, Т. 17, № 2, 17-35. doi: 10.21702/rpj.2020.2.2

МЕДИЦИНСКАЯ ПСИХОЛОГИЯ

в показателях наследуемости подкорковых структур, желудочков мозга, характеристик мозолистого тела, объема мозжечка и др.

В целом генетический анализ структурных характеристик мозга показывает, что умеренные показатели наследуемости (20 до 50 \%) характерны для небольшого ряда структур (например, проводящие пути кортикоспинального тракта или объем боковых желудочков), тогда как наследуемость большинства показателей - более 50 \% (Strike et al., 2015). Построение многомерных близнецовых моделей позволяет также оценить выраженность генетических корреляций, показывающих вклад генетических факторов в ковариацию между показателями на фенотипическом уровне. Так, для структур мозга наблюдаются выраженные генетические корреляции между характеристиками серого и белого вещества различных участков коры головного мозга: корреляции от 30 до $60 \%$ - для связи затылочных областей мозга с остальными отделами коры, от 80 до $95 \%$ - для объема серого и белого вещества во фронтальных, теменных и височных областях (Schmitt et al., 2008). Для подкорковых структур мозга выявлен общий генетический фактор, который объясняет до $60 \%$ фенотипической ковариации (Schmitt et al., 2010). Наличие высоких генетических корреляций между характеристиками различных структур мозга может указывать на то, что одни и те же генетические факторы могут быть связаны с вариабельностью широкого ряда характеристик в мозге.

\section{Возрастные изменения в наследуемости структурных характеристик мозга}

Как известно, возрастные изменения мозга происходят на протяжении всей жизни человека. Так, МРТ-исследования в широком возрастном диапазоне (от 4 до 20 лет) показали увеличение объема серого и белого вещества в период от 4 лет до раннего подросткового возраста. В раннем подростковом возрасте объем серого вещества начинает снижаться (кроме серого вещества в височной области, объем которого увеличивается вплоть до позднего подросткового возраста), тогда как объем белого вещества продолжает расти (Paus et al., 1999; Durston et al., 2001). К шестилетнему возрасту общий объем мозга ребенка достигает 95 \% объема мозга взрослого человека (Paus et al., 1999). Таким образом, несмотря на то, что к 6 годам общий размер мозга практически достигает своего постоянного объема, соотношение серого и белого вещества изменяется в период с подросткового до зрелого возраста (Paus, 2005; Toga \& Thompson, 2005; Raz et al., 2004). В зрелом возрасте наблюдается уменьшение общего объема мозга, связанное с уменьшением объема серого вещества (Bartzokis et al., 2001). В то же время объем белого вещества продолжает возрастать до 45 лет; интересно, что у части выборки здоровых испытуемых общий объем мозга увеличивается вплоть до 40-летнего возраста (van Haren et al., 2008). В целом во взрослом возрасте продолжаются значительные изменения структурных особенностей мозга: продолжается увеличение объема белого вещества, наблюдается нелинейный характер уменьшения общего объема мозга, а также объема и плотности серого вещества.

Психогенетические исследования показывают, что генетические влияния могут различаться в разных возрастах. Так, например, наследуемость интеллекта увеличивается в ходе развития (Plomin \& Craig, 1997; Haworth et al., 2009). Соотношение генетических и средовых факторов изменчивости мозговых структур также может изменяться в ходе онтогенеза. Показатель наследуемости общего объема мозга в первый год жизни составляет 69 \% (Gilmore et al., 2010), к 9 годам увеличиваясь до 94 \% (Peper et al., 2009), и к 12 годам - до 96 \% (van Soelen et al., 2010). Такие высокие значения наблюдаются до 27-летнего возраста (Bartley et al., 1997) 
ЗАХАРОВ И. М., МАЛЫХ С. Б.

ИССЛЕДОВАНИЯ СТРУКТУРНЫХ ХАРАКТЕРИСТИК МОЗГА В ПСИХОГЕНЕТИКЕ

РосСИЙскИй психологИЧЕСКИй ЖУРнАл, 2020, Т. 17, № 2, 17-35. doi: 10.21702/rpj.2020.2.2

МЕДИЦИНСКАЯ ПСИХОЛОГИЯ

и уменьшаются к 30 годам до 90 \% (Baaré et al., 2001), к 49 годам - до $69 \%$ (Winkler et al., 2010), и к 64 годам - до 46 \% (DeStefano et al., 2009). Генетический вклад в вариабельность размера мозжечка также увеличивается с 70 \% в 8 лет (Yoon et al., 2011) до 89 \% в 11 (Wallace et al., 2006) и $96 \%$ в 12 лет (van Soelen et al., 2012). После максимума в 12 лет наблюдается уменьшение роли генетических факторов: в 17 лет - до 80 \% (Bartley et al., 1997), и до 52 \% в 47 (Batouli, Trollor, Wen, \& Sachdev, 2014). Сходная картина наблюдается и для показателей наследуемости объема серого и белого вещества, внутричерепного объема, отдельных долей мозга, объема желудочков. Обобщенные результаты исследований приведены на рисунке 1.

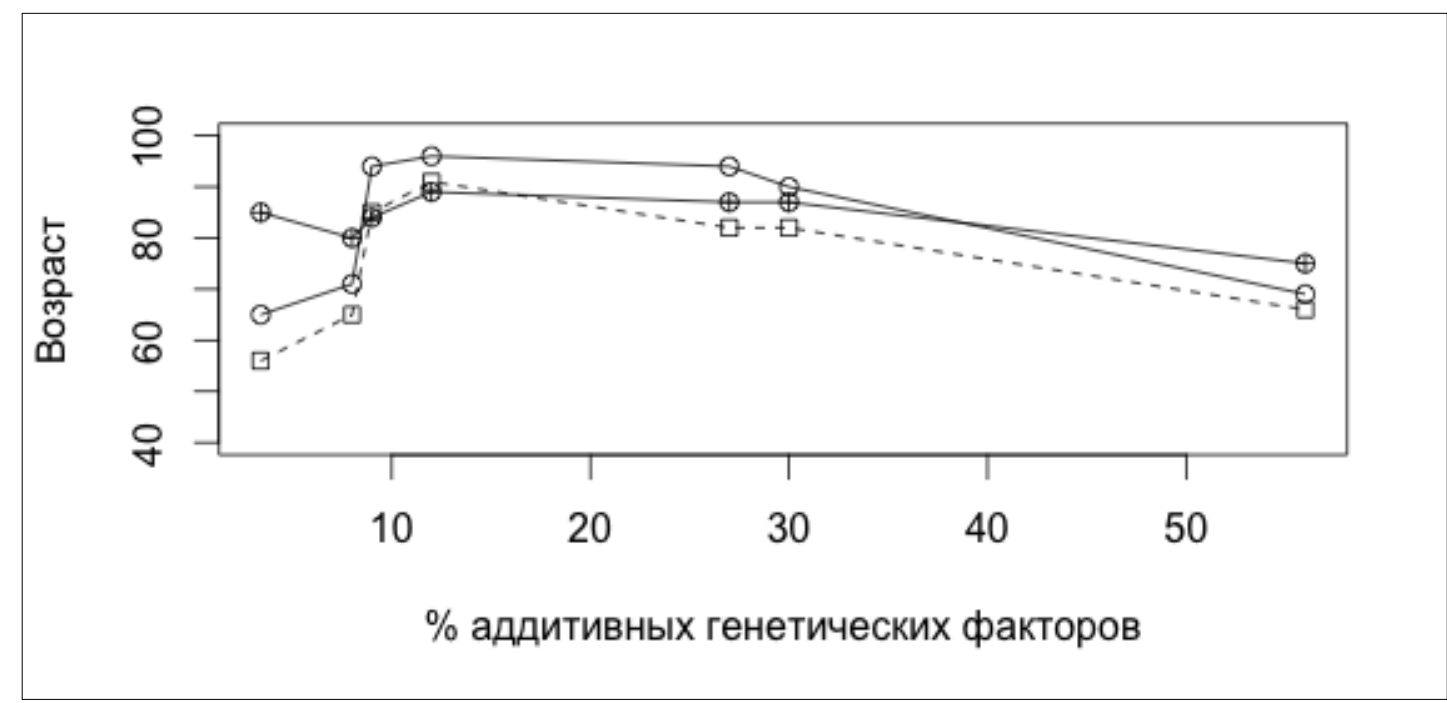

Рисунок 1. Изменение вклаАа аАлитивных генетических фракторов в вариабельность общего объема мозга, объема серого и белого вещества (алаптировано на основе $а$ анных Batouli et al., 2014)

Условные обозначения: круглые полые точки -общий объем мозга; квадратные точки-объем серого вещества; круглые точки с крестом - объем белого вещества.

Исследования с применением метода ДТТ на основании оценки фракционной анизотропии (характеризующей количество и ориентации проводящих путей (трактов) белого вещества головного мозга) также показали наличие возрастных различий. Так, масштабное генетически информативное исследование (705 близнецов и их сиблингов, подростков и взрослых) выявило, что вклад генетических факторов в вариативность характеристик белого вещества у подростков был более выражен, чем у взрослых (Chiang et al., 2011).

При этом необходимо отметить, что на данный момент результаты исследований объема других структур мозга в разные годы жизни противоречивы, что, возможно, связано с ошибками измерения из-за небольшого размера изучаемых областей (Batouli et al., 2014).

\section{Молекулярно-генетические исследования структурных характеристик мозга}

Несмотря на то, что вклад генетических факторов в индивидуальные различия в большом ряде структурных характеристик мозга установлен в целой серии близнецовых и семейных 
ЗАХАРОВ И. М., МАЛЫХ С. Б.

ИССЛЕДОВАНИЯ СТРУКТУРНЫХ ХАРАКТЕРИСТИК МОЗГА В ПСИХОГЕНЕТИКЕ

РосСИЙСКИЙ пСИХОЛОГИЧЕСКИЙ ЖУРнАл, 2020, Т. 17, № 2, 17-35. doi: 10.21702/rpj.2020.2.2

МЕДИЦИНСКАЯ ПСИХОЛОГИЯ

исследований, конкретные молекулярно-генетические механизмы, которые вносят вклад в эту изменчивость, до сих пор остаются не до конца изученными (Brans et al., 2008). В настоящее время все больше генетически информативных исследований направлено на идентификацию конкретных генов, вносящих вклад в вариативность нейрофизиологических особенностей. Эти особенности связаны с большим количеством генетических факторов с малыми размерами эффектов (так называемые локусы количественных признаков - Quantitative Trait Loci). Ha ceгодняшний день разработаны методы, которые позволяют провести картирование участков генома, связанных с интересующими исследователей количественными признаками при помощи молекулярных маркеров (таких как, например, однонуклеотидные полиморфизмы, ОНП). Такой метод получил название метода анализа генетических ассоциаций, целью которого является определение ассоциаций между одним или множеством ДНК-полиморфных вариантов и каким-либо (например, нейрофизиологическим) признаком (Малых и др., 2016).

Один из подходов к анализу генетических ассоциаций - это подбор генов-кандидатов, выделяемых на основе теоретических биологических механизмов формирования исследуемых признаков. На сегодняшний день выявлен целый ряд генов-кандидатов, связанных с различиями в структурных характеристиках мозга. Среди наиболее исследуемых генов можно назвать, например, BDNF, APOE, MECP2, HFE, MTFHR, NRG1.

Нейротрофический фактор BDNF (brain-derived neurotrophic factor) играет важнейшую роль в формировании центральной нервной системы, участвуя в процессах пролиферации и синаптического роста нейронов мозга, а также в модуляции синаптических сигналов (например, в случае долговременной потенциации нейронов гиппокампа). Замены валина на метионин в кодоне 66 гена BDNF (аллеля Val66Met) связывают с изменениями структурных и функциональных характеристик гиппокампа, которые, в свою очередь, влияют на показатели рабочей памяти у человека. Другой важный участник метаболических процессов, связанных с ростом, дегенерацией и регенерацией нервных клеток - аполипротеин плазмы крови APoE. Он связан с нормальным катаболизмом богатых триглицеридами составляющими липопротеинов. Было показано, что аллель АроE4 связан с генетическими рисками развития болезни Альцгеймера (Lu et al., 2011). Разные варианты гена MTHFR связывают с патологическим развитием нервной трубки. Существуют аллели MTHFR, которые ассоциируются с повышенным уровнем гомоцистеина в плазме крови, а также повреждениями нервной ткани и атрофией мозга (Brans et al., 2008). Ген HFE регулирует абсорбцию ионов железа.

B работе Jahanshad et al. (2012) было показано, что с вариациями в этом гене связана целостность нервных волокон, измеренная с помощью фракционной анизотропии ДТВ. Эти данные могут указывать на важную роль метаболизма ионов железа в развитии и дегенерации нервной ткани. Ген МЕСР2 кодирует белок, связывающийся с метилированной ДНК, и участвует в процессах дифференциации клеток во время эмбрионального развития. Joyner et al. (2009) показали наличие ассоциации между этим геном и площадью поверхности (но не толщиной) коры головного мозга. Ген нейрегулина (NRG1) и ген рецептора нейрегулина (ErbB4) связывают с широким спектром проявлений патогенеза у больных шизофренией и биполярным расстройством. Предполагается, что на структурном уровне эти процессы ассоциируются с изменениями в целостности белого вещества в переднем слое внутренней капсулы (anterior limb of the internal capsule, ALIC). Изменения в сигнальном пути NRG1-ErbB4, выявленные у групп испытуемых с разными симптомами, кодируются аллелями s4673628 (Zuliani et al., 2011), rs6994992 (Mclntosh et al., 2007), rs839523 (Konrad et al., 2009). 
ЗАХАРОВ И. М., МАЛЫХ С. Б.

ИССЛЕДОВАНИЯ СТРУКТУРНЫХ ХАРАКТЕРИСТИК МОЗГА В ПСИХОГЕНЕТИКЕ

РоссиЙский псИХологИЧЕСКИЙ ЖУРнАл, 2020, Т. 17, № 2, 17-35. doi: 10.21702/rpj.2020.2.2

МЕДИЦИНСКАЯ ПСИХОЛОГИЯ

Вероятно, что структурные характеристики мозга связаны с суммарным действием большого количества генов. В качестве примера можно привести данные о генах, которые вносят наибольший вклад в вариабельность объема височной коры (Kohannim et al., 2012).

При изучении структурных характеристик мозга большое количество изучаемых генов приводит к проблеме множественных сравнений и большой вероятности ошибок I рода. Для решения этой проблемы используются специальные поправки и статистические процедуры (например, Family-Wise Error Rate - FWER, Worsley et al., 1996; и False Discovery Rate - FDR, Genovese, Lazar, \& Nichols, 2002). Моделирование на основе реальных генетических данных показывает, что применение поправок на множественные сравнения позволяет снизить количество ошибок I рода до 5 \% (Meyer-Lindenberg et al., 2008). Однако все равно сохраняется необходимость репликации полученных данных в независимых исследованиях. Так, например, в ряде исследований была обнаружена связь гена FOXP2, ассоциируемого с развитием речи у людей в эволюции, с характеристиками разных структур мозга (в первую очередь, нижней передней извилины, хвостатого ядра, мозжечка). Однако первоначальные данные были получены в МРТ-исследованиях на группах от 14 до 96 испытуемых. Тем не менее, репликация данных на выборке из 1301 человека не выявила связи вариабельности нейроанатомических показателей с OHП FOXP2 (Hoogman et al., 2014). Существуют эволюционные и экспериментальные предпосылки считать гены, связанные с первичной микроцефалией (ASPM, MCPH1, CDK5RAP2 и BRCA1 гены), основой для индивидуальных различий в размерах мозга в нормальной популяции, а также выраженности лингвистических и когнитивных способностей. Тем не менее, в исследовании 1776 испытуемых из 789 близнецовых семей не было обнаружено связей между этими генами и исследуемыми признаками (Bates et al., 2008).

В связи с определенными ограничениями метода генов-кандидатов, в последние годы в генетически информативных исследованиях структуры мозга используется метод полногеномного анализа ассоциаций (Genome-Wide Association Study, GWAS), который рассматривает сотни тысяч ДНК-маркеров одновременно в их взаимосвязи с различными признаками. GWAS-исследования фокусируются, в первую очередь, на изучении однонкулеотидных полиморфизмов (ОНП) - типа вариабельности в последовательности ДНК у разных людей, связанного с заменой одного нуклеотида на другой в конкретном ДНК-локусе (например, аденина (A) на тимин (Т)) и характеризующегося наличием разных аллелей (обычно двух) в популяции (например, аллель А и аллель Т). Учитывая величину исследуемых эффектов, для получения статистически значимых результатов требуются большие выборки испытуемых.

Наиболее полный на сегодняшний день метаанализ связей структурных характеристик мозга с конкретными генами был проведен коллективом исследователей из консорциума ENIGMA (Thompson et al., 2020). В первоначальной работе консорциума анализировались данные 30717 испытуемых из 50 когорт. Вся выборка была поделена на 2 равных части для проведения полногеномного (GWAS) анализа ассоциаций отдельных локусов со структурными характеристиками отделов мозга. Математическая модель ассоциаций была проверена на подвыборке из 13171 испытуемого и верифицирована на второй подвыборке из 17546 испытуемых. В работе были обнаружены 8 основных локусов, связанных с объемом таких структур, как хвостатое ядро, гиппокамп, скорлупа чечевицеобразного ядра, а также с внутричерепным объемом мозга. Значимые связи, выявленные в исследовании, объясняли от 0.17 до $0.52 \%$ фенотипической вариабельности объемов в различных областях мозга. Интересно, что проанализированные локусы ассоциировались с отдельными структурами, а не с множественными эффектами 
ЗАХАРОВ И. М., МАЛЫХ С. Б.

ИССЛЕДОВАНИЯ СТРУКТУРНЫХ ХАРАКТЕРИСТИК МОЗГА В ПСИХОГЕНЕТИКЕ

РосСИЙСКИЙ пСИХОЛОГИЧЕСКИЙ ЖУРнАл, 2020, Т. 17, № 2, 17-35. doi: 10.21702/rpj.2020.2.2

МЕДИЦИНСКАЯ ПСИХОЛОГИЯ

на разные участки мозга. Размер эффекта гена KTN1 был максимальным для различий в объеме скорлупы мозга. Этот ген кодирует белок рецептора кинектина, который связывает кинезин, а также вовлечен в процессы транспорта органелл. Экспрессия гена DCC в мозге наиболее выражена во время первых двух триместров пренатального развития, что предполагает его связь с регуляцией объема мозговых структур на ранних этапах роста нервной системы. Как известно, ген DCC (rs62097986; 18q21.2; $\mathrm{n}=28,036 ; \mathrm{P}=1.01$ * 10-13) кодирует нетрин (рецептор, связанный с ростом и миграцией аксона, а также с ростом стриатума). Экспрессия BCL2L1 максимальна на 24-38 неделях после зачатия, в период наиболее активного апоптоза в скорлупе мозга. BCL2L1 (rs6087771; 20q11.21; $\mathrm{n}=25,540 ; \mathrm{P}=1.28$ * 10-12) кодирует антиапоптоз-фактор, тормозящий программируемую смерть незрелых нейронов в мозге. Максимально DLG2 активен в середине внутриутробного развития во время роста стриатума. Ген DLG2 (rs683250; $11 q 14.1 ; n=26,258 ; \mathrm{P}=3.94$ * 10-11) кодирует плотность протеина 93 в постинаптической области (Hibar et al., 2015). Генетические варианты в этом локусе ассоциируют с процессами научения и когнитивной гибкостью (в некоторых исследованиях также с шизофренией).

В более поздних исследованиях данные, собранные в рамках консорциума ENIGMA, были объединены с данными двух других масштабных проектов: консорциума CHARGE и биобанка Великобритании (UK Biobank). На основании проведенного анализа исследователи смогли выявить более 200 индивидуальных локусов, вносящих вклад в вариабельность характеристик 70 разных структур мозга (Thompson et al., 2020). При этом, несмотря на то, что вклад единичного локуса в общую фенотипическую вариабельность варьировался в диапазоне от 0,1\% до 1\%, объединение обнаруженных локусов в одну модель позволяло объяснить до $20 \%$ общей дисперсии. В целом для генетических локусов, связанных с морфологией мозга, наблюдается связь с регуляторными генами, а также с регуляторными элементами, специфическими для человека по сравнению с другими млекопитающими. Для доступа к полученным данным коллективом ENIGMA на сегодняшний день создан интерактивный сайт https://enigma-brain. org/enigmavis/, позволяющий исследователям получить более детальную информацию как об интересующих их структурах мозга, так и о вкладе конкретных генетических локусов.

\section{Результаты и их обсуждение}

\section{Структурные характеристики мозга как эндофенотипы поведенческих фенотипов}

В рамках концепции эндофенотипов структурные характеристики мозга могут рассматриваться как в качестве промежуточных признаков, связанных с психическими заболеваниями, так и в контексте изучения нормальных психологических признаков. Так, например, согласно современным метааналитическим исследованиям, общий объем мозга, объем серого и белого вещества связаны с общим интеллектом (Gignac \& Bates, 2017). В исследованиях связи интеллекта и общего объема серого и белого вещества в мозге наблюдаются умеренные $(r= \pm 0.30)$ фенотипические корреляции. Использование в генетически информативных исследованиях многомерных моделей позволяет увидеть, что существует общий генетический вклад в таких показателях, как индивидуальные различия в объеме мозга и баллы тестов на вербальный и невербальный интеллект (Posthuma, 2002; Малых и др., 2016), а также в ковариации объема серого вещества лобных долей и интеллекта (Strike et al., 2015; Carmelli et al., 2002). Кроме того, выявленные общие генетические факторы наблюдаются в ковариации вербального и невербального интеллекта с такими структурами головного мозга, как серое вещество 
ЗАХАРОВ И. М., МАЛЫХ С. Б.

ИССЛЕДОВАНИЯ СТРУКТУРНЫХ ХАРАКТЕРИСТИК МОЗГА В ПСИХОГЕНЕТИКЕ

РосСИйский психологИЧЕСКИй жУРнАл, 2020, Т. 17, № 2, 17-35. doi: 10.21702/rpj.2020.2.2

МЕДИЦИНСКАЯ ПСИХОЛОГИЯ

лобных, затылочных и парагиппокампальных областей, а также волокон белого вещества верхнего затылочно-лобного пучка и мозолистого тела (Hulshoff Pol et al., 2006). При этом близнецовые исследования также позволяют получить информацию о роли средовых факторов в индивидуальных различиях признака. Например, общие средовые влияния обнаружены для индивидуальных различий в характеристиках управляющих функций и объеме лобно-височных областей мозга (Carmelli et al., 2002). При этом необходимо учитывать, что воздействие средовых и генетических факторов может быть разнонаправленным, тем самым снижая фенотипические корреляции (Strike et al., 2015).

B таблице 1 приведены результаты основных генетически информативных исследований ковариации интеллекта и структурных характеристик мозга.

\begin{tabular}{|c|c|c|c|c|c|c|}
\hline \multicolumn{7}{|c|}{$\begin{array}{l}\text { Таблица } 1 \\
\text { Генетически инорормативные исслеАования связ } \\
\text { мозга и общих когнитивных способностей (IQ) }\end{array}$} \\
\hline Ковариация френотипов & $\frac{\text { Выборка }}{\text { M3/А3 }}$ & Bo3pact & $\underline{r_{p}}$ & $\underline{r}_{q}$ & $\underline{r}_{\underline{e}}$ & Статья \\
\hline Общий объем коры и FSIQ & $25 / 23$ & $>12 \wedge е т$ & - & 0.48 & - & $\begin{array}{l}\text { Pennigton } \\
\text { et al., } 2000\end{array}$ \\
\hline $\begin{array}{l}\text { Объем серого вещества } \\
\text { коры ГМ и фоктор g } \\
\text { Объем белого вещества } \\
\text { коры ГМ и фрактор g }\end{array}$ & $24 / 31$ & $19-69$ & $\begin{array}{l}0.25 \\
0.24\end{array}$ & $\begin{array}{l}0.29 \\
0.24\end{array}$ & - & $\begin{array}{c}\text { Posthuma, } \\
2002\end{array}$ \\
\hline $\begin{array}{l}\text { Белое вещество } \\
\text { мозолистого тела }\end{array}$ & & & & & & \\
\hline $\begin{array}{l}\text { и вербальный } \\
\text { интеллект (VIQ) } \\
\text { Парагиппокампальное } \\
\text { белое вещество и РIQ }\end{array}$ & $54 / 58$ & $19-69$ & $\begin{array}{l}0.14 \\
0.23\end{array}$ & $\begin{array}{l}0.15 \\
0.40\end{array}$ & - & $\begin{array}{l}\text { Hulshoff Pol } \\
\text { et al., } 2006\end{array}$ \\
\hline $\begin{array}{l}\text { Объем серого вещества } \\
\text { коры и прогрессивные } \\
\text { матрицы Равена }\end{array}$ & $48 / 64$ & 9 & 0.22 & 0.36 & -0.16 & $\begin{array}{l}\text { van } \\
\text { Leeuwen } \\
\text { et al., } 2009\end{array}$ \\
\hline $\begin{array}{l}\text { Толщина коры в передних } \\
\text { меАиальных областях } \wedge П \\
\text { и заты^очных областях ПП } \\
\text { и FSIQ }\end{array}$ & $77 / 84$ & 30 & $\begin{array}{c}0.8 \\
0.34\end{array}$ & $\begin{array}{c}0.56 \\
1\end{array}$ & $\begin{array}{r}-0.66 \\
0.22\end{array}$ & $\begin{array}{l}\text { Brans et al. } \\
2010\end{array}$ \\
\hline
\end{tabular}


ЗАХАРОВ И. М., МАЛЫХ С. Б.

ИССЛЕДОВАНИЯ СТРУКТУРНЫХ ХАРАКТЕРИСТИК МОЗГА В ПСИХОГЕНЕТИКЕ

РосСИЙСКИЙ пСИХОЛОГИЧЕСКИЙ ЖУРнАл, 2020, Т. 17, № 2, 17-35. doi: 10.21702/rpj.2020.2.2

МЕДИЦИНСКАЯ ПСИХОЛОГИЯ

\begin{tabular}{|c|c|c|c|c|c|c|}
\hline $\begin{array}{l}\text { Таблица } 1 \\
\text { Генетически инорорматив } \\
\text { мозга и общих когнитивны }\end{array}$ & $\begin{array}{l}\text { е исслеАОв } \\
\text { пособност }\end{array}$ & $\begin{array}{l}\text { ия Связи С } \\
\text { (IQ) }\end{array}$ & Ктурнь & каракте & 1Стик ГС & рвного \\
\hline Ковариация оренотипов & $\frac{\text { Выборка }}{\underline{M 3 / \Delta 3}}$ & Bозраст & $\underline{r} \underline{p}$ & $\underline{r}_{g}$ & $\underline{r}_{\underline{e}}$ & Статья \\
\hline $\begin{array}{l}\text { Общий объем мозга } \\
\text { и FSIQ } \\
\text { Объем белого вещества } \\
\text { коры ГM и VIQ }\end{array}$ & $11 / 21$ & $19-56$ & $\begin{array}{l}0.27 \\
0.29\end{array}$ & $\begin{array}{c}0.50 \\
1\end{array}$ & $\begin{array}{l}-0.17 \\
-0.41\end{array}$ & $\begin{array}{c}\text { Brouwer et } \\
\text { al., } 2014\end{array}$ \\
\hline $\begin{array}{l}\text { Толщина коры в области } \\
\text { околоцентральной Аольки } \\
\text { ^П и FSIQ } \\
\text { Толщина коры в области } \\
\text { К^ина и VIQ }\end{array}$ & $23 / 28$ & 12 & $\begin{array}{l}-0.29 \\
-0.28\end{array}$ & $\begin{array}{c}-0.32 \\
-1\end{array}$ & $\begin{array}{r}-0.24 \\
0.39\end{array}$ & $\begin{array}{l}\text { Brouwer } \\
\text { et al., } 2014\end{array}$ \\
\hline $\begin{array}{l}\text { Объем скорлупы (бил.) } \\
\text { и таламуса (бил.) и FSIQ }\end{array}$ & $50 / 56$ & $19-55$ & $\begin{array}{l}0.01 \\
0.26\end{array}$ & $\begin{array}{c}0 \\
0.29\end{array}$ & $\begin{array}{l}0.08 \\
0.08\end{array}$ & $\begin{array}{l}\text { Bohlken } \\
\text { et al., } 2014\end{array}$ \\
\hline $\begin{array}{l}\text { Толщина серого вещество } \\
\text { и площадь поверхности } \\
\text { коры ГМ и общие } \\
\text { когнитивные способности }\end{array}$ & $131 / 96$ & $51-60$ & $\begin{array}{l}0.08 \\
0.21\end{array}$ & $\begin{array}{l}0.09 \\
0.24\end{array}$ & $\begin{array}{l}0.10 \\
0.21\end{array}$ & $\begin{array}{l}\text { Vuoksimaa } \\
\text { et al., } 2015\end{array}$ \\
\hline
\end{tabular}

Кроме генетических и средовых факторов самих по себе, важную роль в формировании индивидуальных различий может играть генотип-средовое взаимодействие. В ряде работ наблюдается взаимодействие социально-экономического статуса (СЭС) и генетических факторов, которые влияют на целостность волокон белого вещества мозга, измеренных с помощью метода диффузной тензорной томографии: показатели наследуемости были выше у участников с более высоким СЭС (Малых и др., 2016). Другим примером генотип-средового взаимодействия можно считать результаты исследований различий в наследуемости показателей ДТТ у участников с разным уровнем IQ. Согласно полученным результатам Blockland et al. (2012), у группы участников с высокими показателями IQ более $80 \%$ наблюдаемых индивидуальных различий в фракционной анизотропии в задней ножке внутренней капсулы, колене мозолистого тела, верхнем лучистом венце и таламусе было связано с генетическими факторами. При этом у участников с низкими показателями IQ вклад генетики не превышал $40 \%$. Несмотря 
ЗАХАРОВ И. М., МАЛЫХ С. Б.

ИССЛЕДОВАНИЯ СТРУКТУРНЫХ ХАРАКТЕРИСТИК МОЗГА В ПСИХОГЕНЕТИКЕ

РосСИйский психологИЧЕСКИй жУРнАл, 2020, Т. 17, № 2, 17-35. doi: 10.21702/rpj.2020.2.2

МЕДИЦИНСКАЯ ПСИХОЛОГИЯ

на то, что конкретные механизмы такого взаимодействия до сих пор неясны и требуют дополнительного изучения, можно предположить, что связь индивидуальных различий в показателях СЭС и интеллекта с наследуемостью структурных характеристик мозга может зависеть от характеристик экспрессии определенных генов. Так, например, известно, что экспрессия гена нейротрофического фактора BDNF, связанного с ростом нейронов и когнитивными функциями, может меняться под воздействием обучения (Kesslak, So, Choi, Cotman, \& GomezPinilla, 1998). Генетические факторы могут также играть активную роль в трансформации физической и социальной среды, что может проявляться в эффекте генно-средовых корреляций (Hoogman et al., 2019). В частности, за счет вклада генов, влияющих на индивидуальные различия в характеристиках белого вещества, может меняться аксональная проводимость нейронных сигналов в таламусе и кортикоспинальном тракте, что может косвенно отражаться на формировании показателей интеллекта. Таким образом, генетические эффекты могут быть опосредованы другими показателями, такими как пол, возраст, СЭС и IQ и дp. (Hackman \& Farah, 2009; Zavala et al., 2018).

Генетически информативные исследования также активно применяются при изучении этиологии психопатологических признаков. В ряде работ, направленных на изучение мозговых механизмов шизофрении, у пациентов было обнаружено прогрессирующее во времени уменьшение объема лобных и височных долей коры больших полушарий и уменьшение общего объема мозга. Оказалось, что в ковариации между рисками развития шизофрении и изменениями в структурных характеристиках мозга выраженную роль играют аддитивные генетические влияния (для общего объема мозга - $66 \%$, лобных долей - $76 \%$, височных долей - 79\%) (Batouli et al., 2014; Малых и др., 2016). Общий генетический фактор, полученный на основании многомерного моделирования структурными уравнениями, также объясняет значительную часть вариативности связей между шизофренией и концентрацией серого вещества в лобной и височной областях (Brans et al., 2008). Снижение объема белого вещества связано с генетическим риском развития биполярного расстройства (77\%), несмотря на то, что конкретные генетические механизмы такой связи требуют дальнейшего анализа (Gershon, Alliey-Rodriguez, \& Liu, 2011). Современные масштабные исследования связи структурных характеристик мозга и психопатологии позволили выявить нейрофизиологические профили подкорковых структур мозга, характерные для лиц с такими заболеваниями, как шизофрения, биполярное расстройство, СДВГ и большое депрессивное расстройство (major depressive disorder, MDD). На основе данных 21199 человек было показано, что шизофрения, биполярное расстройство и большое депрессивное расстройство связаны со снижением объема гиппокампальных областей мозга, желудочков мозга, таламуса, амигдалы и прилежащего ядра, при этом выраженность изменений максимальна для шизофрении, приблизительно вдвое меньше для биполярного расстройства и вчетверо меньше для большого депрессивного расстройства (Mufford et al., 2019). Для СДВГ также характерны изменения амигдалы и прилежащего ядра, при этом также наблюдается уменьшение объема скорлупы мозга и хвостатого ядра (более подробные данные представлены на интерактивном сайте проекта ENIGMA).

В связи с переходом к формату многоцентровых консорциумов, у современных исследователей открывается новое поле возможностей. Так, за счет увеличения изучаемых выборок, были получены данные на новых группах психопатологии, таких как синдром Туретта (Mufford et al., 2019), бессонница (Grasby et al., 2020) или анорексия (Walton et al., 2019). Кроме того, к исследованиям ОНП добавились исследования вариаций в числе копий генов (Сopy Number 
ЗАХАРОВ И. М., МАЛЫХ С. Б.

ИССЛЕДОВАНИЯ СТРУКТУРНЫХ ХАРАКТЕРИСТИК МОЗГА В ПСИХОГЕНЕТИКЕ

РосСИЙСКИЙ пСИХОЛОГИЧЕСКИЙ ЖУРнАл, 2020, Т. 17, № 2, 17-35. doi: 10.21702/rpj.2020.2.2

МЕДИЦИНСКАЯ ПСИХОЛОГИЯ

Variations) в качестве факторов риска развития психопатологии и индивидуальных различий в характеристиках как корковых, так и подкорковых структур мозга (Thompson et al., 2020). В настоящее время также наблюдается постепенный переход от полногеномных исследований к всегеномным, в рамках которых возможен учет даже редко встречающихся генетических вариантов (Medland, Jahanshad, Neale, \& Thompson, 2014).

\section{Заключение}

Увеличение количества генетически информативных исследований структур и функций головного мозга, а также значительное увеличение изучаемых выборок являются одними из важнейших трендов в исследованиях на стыке генетики и нейронауки в последние годы. Результаты близнецовых исследований убедительно свидетельствуют о наличии значительной роли генетических факторов в формировании индивидуальных различий во многих структурных характеристиках мозга (общий объем мозга, толщина и площадь поверхности коры головного мозга, объем серого и белого вещества в мозге и др.). При этом развитие молекулярно-генетических методов позволяет приблизиться к анализу связи нейрофизиологических характеристик с конкретными полиморфными вариантами различных генов-кандидатов. Комплексный подход к изучению позволяет прослеживать путь от генов к психологическим фенотипам через опосредующие их индивидуальные различия в структурных и функциональных особенностях головного мозга. Переход к новым типам молекулярно-генетических исследований, в том числе к исследованиям таких генетических факторов, как вариация числа копий генов, полногеномным и всегеномным (whole-genome) исследованиям, открывает новые возможности для определения молекулярно-генетических механизмов, лежащих в основе индивидуальных различий в структурных характеристиках мозга и психологических признаках.

\section{Литература}

Малых, С. Б., Егорова, М. С. и Мешкова, Т. А. (2008). Психогенетика. Санкт-Петербург: Питер. Малых, С. Б., Ковас, Ю. В. и Гайсина, Д. А. (ред.) (2016). Геномика поведения: детское развиmие и образование. Томск: Издательский Дом Томского государственного университета.

Adrián-Ventura, J., Costumero, V., Parcet, M. A., \& Ávila, C. (2019). Linking personality and brain anatomy: A structural MRI approach to Reinforcement Sensitivity Theory. Social Cognitive and Affective Neuroscience, 14(3), 329-338. doi: 10.1093/scan/nsz011

Baaré, W. F. C., Hulshoff Pol, H. E., Boomsma, D. I., Posthuma, D., de Geus, E. J. C., Schnack, H. G., ... Kahn, R. S. (2001). Quantitative genetic modeling of variation in human brain morphology. Cerebral Cortex, 11(9), 816-824. doi: 10.1093/cercor/11.9.816

Bartley, A. J., Jones, D. W., \& Weinberger, D. R. (1997). Genetic variability of human brain size and cortical gyral patterns. Brain, 120(2), 257-269. doi: 10.1093/brain/120.2.257

Bartzokis, G., Beckson, M., Lu, P. H., Nuechterlein, K. H., Edwards, N., \& Mintz, J. (2001). Agerelated changes in frontal and temporal lobe volumes in men. Archives of General Psychiatry, 58(5), 461-465. doi: 10.1001/archpsyc.58.5.461

Basser, P. J., Mattiello, J., \& Lebihan, D. (1994). Estimation of the effective self-diffusion tensor from the NMR spin echo. Journal of Magnetic Resonance, Series B, 103(3), 247-254. doi: 10.1006/ jmrb.1994.1037 
ЗАХАРОВ И. М., МАЛЫХ С. Б.

ИССЛЕДОВАНИЯ СТРУКТУРНЫХ ХАРАКТЕРИСТИК МОЗГА В ПСИХОГЕНЕТИКЕ

РоссиЙский псИХологИЧЕСКИЙ ЖУРнАл, 2020, Т. 17, № 2, 17-35. doi: 10.21702/rpj.2020.2.2

МЕДИЦИНСКАЯ ПСИХОЛОГИЯ

Bates, T. C., Luciano, M., Lind, P. A., Wright, M. J., Montgomery, G. W., \& Martin, N. G. (2008). Recently-derived variants of brain-size genes ASPM, MCPH1, CDK5RAP and BRCA1 not associated with general cognition, reading or language. Intelligence, 36(6), 689-693. doi: 10.1016/j. intell.2008.04.001

Batouli, S. A. H., Trollor, J. N., Wen, W., \& Sachdev, P. S. (2014). The heritability of volumes of brain structures and its relationship to age: A review of twin and family studies. Ageing Research Reviews, 13, 1-9. doi: 10.1016/i.arr.2013.10.003

Blokland, G. A. M., de Zubicaray, G. I., McMahon, K. L., \& Wright, M. J. (2012). Genetic and environmental influences on neuroimaging phenotypes: A meta-analytical perspective on twin imaging studies. Twin Research and Human Genetics, 15(3), 351-371. doi: 10.1017/thg.2012.11

Bohlken, M. M., Brouwer, R. M., Mandl, R. C. W., van Haren, N. E. M., Brans, R. G. H., van Baal, G. C. M., ... \& Hulshoff Pol, H. E. (2014). Genes contributing to subcortical volumes and intellectual ability implicate the thalamus. Human Brain Mapping, 35(6), 2632-2642. doi: 10.1002/hbm.22356

Brans, R. G. H., Kahn, R. S., Schnack, H. G., van Baal, G. C. M., Posthuma, D., van Haren, N. E. M., ... \& Hulshoff Pol, H. E. (2010). Brain plasticity and intellectual ability are influenced by shared genes. Journal of Neuroscience, 30(16), 5519-5524. doi: 10.1523/JNEUROSCI.5841-09.2010

Brans, R. G. H., van Haren, N. E. M., van Baal, G. C. M., Schnack, H. G., Kahn, R. S., \& Hulshoff Pol, H. E. (2008). Heritability of changes in brain volume over time in twin pairs discordant for schizophrenia. Archives of General Psychiatry, 65(11), 1259-1268. doi: 10.1001/archpsyc.65.11.1259

Brouwer, R. M., Hedman, A. M., van Haren, N. E., Schnack, H. G., Brans, R. G. H., Smit, D. J. A., ... \& Hulshoff Pol, H. E. (2014). Heritability of brain volume change and its relation to intelligence. Neurolmage, 100, 676-683. doi: 10.1016/j.neuroimage.2014.04.072

Brun, C. C., Leporé, N., Pennec, X., Lee, A. D., Barysheva, M., Madsen, S. K., ... Thompson, P. M. (2009). Mapping the regional influence of genetics on brain structure variability - A Tensor-Based Morphometry study. Neurolmage, 48(1), 37-49. doi: 10.1016/j.neuroimage.2009.05.022

Cannon, T. D., Thompson, P. M., van Erp, T. G. M., Huttunen, M., Lonnqvist, J., Kaprio, J., \& Toga, A. W. (2006). Mapping heritability and molecular genetic associations with cortical features using probabilistic brain atlases: Methods and applications to schizophrenia. Neuroinformatics, 4, 5-19. doi: 10.1385/ni:4:1:5

Carmelli, D., DeCarli, C., Swan, G. E., Jack, L. M., Reed, T., Wolf, P. A., \& Miller, B. L. (1998). Evidence for genetic variance in white matter hyperintensity volume in normal elderly male twins. Stroke, 29(6), 1177-1181. doi: 10.1161/01.str.29.6.1177

Carmelli, D., Swan, G. E., DeCarli, C., \& Reed, T. (2002). Quantitative genetic modeling of regional brain volumes and cognitive performance in older male twins. Biological Psychology, 61(1-2), 139-155. doi: 10.1016/s0301-0511(02)00056-x

Chiang, M.-C., Barysheva, M., Shattuck, D. W., Lee, A. D., Madsen, S. K., Avedissian, C., ... Thompson, P. M. (2009). Genetics of brain fiber architecture and intellectual performance. Journal of Neuroscience, 29(7), 2212-2224. doi: 10.1523/jneurosci.4184-08.2009

Chiang, M.-C., McMahon, K. L., de Zubicaray, G. I., Martin, N. G., Hickie, I., Toga, A. W., ... Thompson, P. M. (2011). Genetics of white matter development: A DTI study of 705 twins and their siblings aged 12 to 29. Neurolmage, 54(3), 2308-2317. doi: 10.1016/j.neuroimage.2010.10.015

DeStefano, A. L., Seshadri, S., Beiser, A., Atwood, L. D., Massaro, J. M., Au, R., ... DeCarli, C. (2009). Bivariate heritability of total and regional brain volumes: The Framingham Study. Alzheimer Disease \& Associated Disorders, 23(3), 218-223. doi: 10.1097/wad.0b013e31819cadd8 
Durston, S., Hulshoff Pol, H. E., Casey, B. J., Giedd, J. N., Buitelaar, J. K., \& Engeland, H. V. (2001). Anatomical MRI of the developing human brain: What have we learned? Journal of the American Academy of Child \& Adolescent Psychiatry, 40(9), 1012-1020. doi: 10.1097/00004583-200109000-00009

Genovese, C. R., Lazar, N. A., \& Nichols, T. (2002). Thresholding of statistical maps in functional neuroimaging using the false discovery rate. Neurolmage, 15(4), 870-878. doi: 10.1006/nimg.2001.1037

Gershon, E. S., Alliey-Rodriguez, N., \& Liu, C. (2011). After GWAS: searching for genetic risk for schizophrenia and bipolar disorder. American Journal of Psychiatry, 168(3), 253-256. doi: 10.1176/appi.ajp.2010.10091340

Geschwind, D. H., Miller, B. L., DeCarli, C., \& Carmelli, D. (2002). Heritability of lobar brain volumes in twins supports genetic models of cerebral laterality and handedness. Proceedings of the National Academy of Sciences, 99(5), 3176-3181. doi: 10.1073/pnas.052494999

Gignac, G. E., \& Bates, T. C. (2017). Brain volume and intelligence: The moderating role of intelligence measurement quality. Intelligence, 64, 18-29. doi: 10.1016/j.intell.2017.06.004

Gilmore, J. H., Schmitt, J. E., Knickmeyer, R. C., Smith, J. K., Lin, W., Styner, M., ... Neale, M. C. (2010). Genetic and environmental contributions to neonatal brain structure: A twin study. Human Brain Mapping, 31(8), 1174-1182. doi: 10.1002/hbm.20926

Glahn, D. C., Curran, J. E., Winkler, A. M., Carless, M. A., Kent Jr., J. W., Charlesworth, J. C., ... Blangero, J. (2012). High dimensional endophenotype ranking in the search for major depression risk genes. Biological Psychiatry, 71(1), 6-14. doi: 10.1016/j.biopsych.2011.08.022

Gottesman, I. I., \& Shields, J. (1972). Schizophrenia and genetics. A Twin Study Vantage Point. New York: Acad. Press.

Grasby, K. L., Jahanshad, N., Painter, J. N., Colodro-Conde, L., Bralten, J., Hibar, D. P., ... Medland, S. E. (2020). The genetic architecture of the human cerebral cortex. Science, 367(6484). doi: $10.1126 /$ science.aay 6690

Hackman, D. A., \& Farah, M. J. (2009). Socioeconomic status and the developing brain. Trends in Cognitive Sciences, 13(2), 65-73. doi: 10.1016/j.tics.2008.11.003

Haworth, C. M. A., Wright, M. J., Luciano, M., Martin, N. G., de Geus, E. J. C., van Beijsterveldt, C. E. M., ... Plomin, R. (2009). The heritability of general cognitive ability increases linearly from childhood to young adulthood. Molecular Psychiatry, 15, 1112-1120. doi: 10.1038/mp.2009.55

Hibar, D. P., Stein, J. L., Renteria, M. E., Arias-Vasquez, A., Desrivières, S., Jahanshad, N., ... \& Medland, S. E. (2015). Common genetic variants influence human subcortical brain structures. Nature, 520, 224-229. doi: 10.1038/nature14101

Hilger, K., Ekman, M., Fiebach, C. J., \& Basten, U. (2017). Intelligence is associated with the modular structure of intrinsic brain networks. Scientific Reports, 7, 16088. doi: 10.1038/s41598-017-15795-7

Hoogman, M., Guadalupe, T., Zwiers, M. P., Klarenbeek, P., Francks, C., \& Fisher, S. E. (2014). Assessing the effects of common variation in the FOXP2 gene on human brain structure. Frontiers in Human Neuroscience, 8, 473. doi: 10.3389/fnhum.2014.00473

Hoogman, M., Muetzel, R., Guimaraes, J. P., Shumskaya, E., Mennes, M., Zwiers, M. P., ... Franke, B. (2019). Brain imaging of the cortex in ADHD: A coordinated analysis of large-scale clinical and population-based samples. American Journal of Psychiatry, 176(7), 531-542. doi: 10.1176/appi.ajp.2019.18091033

Hulshoff Pol, H. E., Schnack, H. G., Posthuma, D., Mandl, R. C. W., Baaré, W. F., van Oel, C., ... Kahn, R. S. (2006). Genetic contributions to human brain morphology and intelligence. Journal of Neuroscience, 26(40), 10235-10242. doi: 10.1523/jneurosci.1312-06.2006 
ЗАХАРОВ И. М., МАЛЫХ С. Б.

ИССЛЕДОВАНИЯ СТРУКТУРНЫХ ХАРАКТЕРИСТИК МОЗГА В ПСИХОГЕНЕТИКЕ

РосСИЙскИй психологИЧЕСКИй жУРнАл, 2020, Т. 17, № 2, 17-35. doi: 10.21702/rpj.2020.2.2

МЕДИЦИНСКАЯ ПСИХОЛОГИЯ

lacono, W. G., Malone, S. M., \& Vrieze, S. I. (2017). Endophenotype best practices. International Journal of Psychophysiology, 111, 115-144. doi: 10.1016/j.ijpsycho.2016.07.516

Jahanshad, N., Kohannim, O., Hibar, D. P., Stein, J. L., McMahon, K. L., de Zubicaray, G. I., ... Thompson, P. M. (2012). Brain structure in healthy adults is related to serum transferrin and the H63D polymorphism in the HFE gene. Proceedings of the National Academy of Sciences, 109(14), E851-E859. doi: 10.1073/pnas.1105543109

Jahanshad, N., Lee, A. D., Barysheva, M., McMahon, K. L., de Zubicaray, G. I., Martin, N. G., ... \& Thompson, P. M. (2010). Genetic influences on brain asymmetry: A DTI study of 374 twins and siblings. Neurolmage, 52(2), 455-469. doi: 10.1016/j.neuroimage.2010.04.236

Joshi, A. A., Leporé, N., Joshi, S. H., Lee, A. D., Barysheva, M., Stein, J. L., ... Thompson, P. M. (2011). The contribution of genes to cortical thickness and volume. NeuroReport, 22(3), 101-105. doi: 10.1097/wnr.0b013e3283424c84

Joyner, A. H., Roddey, J. C., Bloss, C. S., Bakken, T. E., Rimol, L. M., Melle, I., ... Dale, A. M. (2009). A common MECP2 haplotype associates with reduced cortical surface area in humans in two independent populations. Proceedings of the National Academy of Sciences, 106(36), 15483-15488. doi: $10.1073 /$ pnas.0901866106

Kesslak, J. P., So, V., Choi, J., Cotman, C. W., \& Gomez-Pinilla, F. (1998). Learning upregulates brainderived neurotrophic factor messenger ribonucleic acid: A mechanism to facilitate encoding and circuit maintenance? Behavioral Neuroscience, 112(4), 1012-1019. doi: 10.1037/0735-7044.112.4.1012

Kochunov, P., Glahn, D. C., Lancaster, J. L., Winkler, A. M., Smith, S., Thompson, P. M., ... Blangero, J. (2010). Genetics of microstructure of cerebral white matter using diffusion tensor imaging. Neurolmage, 53(3), 1109-1116. doi: 10.1016/j.neuroimage.2010.01.078

Kohannim, O., Hibar, D. P., Stein, J. L., Jahanshad, N., Hua, X., Rajagopalan, P., ... The Alzheimer's Disease Neuroimaging Initiative (2012). Discovery and replication of gene influences on brain structure using LASSO regression. Frontiers in Neuroscience, 6, 115. doi: $10.3389 /$ fnins.2012.00115

Konrad, A., Vucurevic, G., Musso, F., Stoeter, P., Dahmen, N., \& Winterer, G. (2009). ErbB4 genotype predicts left frontotemporal structural connectivity in human brain. Neuropsychopharmacology, 34, 641-650. doi: 10.1038/npp.2008.112

Lenroot, R. K., Schmitt, J. E., Ordaz, S. J., Wallace, G. L., Neale, M. C., Lerch, J. P., ... Giedd, J. N. (2009). Differences in genetic and environmental influences on the human cerebral cortex associated with development during childhood and adolescence. Human Brain Mapping, 30(1), 163-174. doi: $10.1002 / \mathrm{hbm} .20494$

Llera, A., Wolfers, T., Mulders, P., \& Beckmann, C. F. (2019). Inter-individual differences in human brain structure and morphology link to variation in demographics and behavior. eLife, 8. doi: 10.7554/elife.44443

Lu, P. H., Thompson, P. M., Leow, A., Lee, G. J., Lee, A., Yanovsky, I., ... Bartzokis, G. (2011). Apolipoprotein $E$ genotype is associated with temporal and hippocampal atrophy rates in healthy elderly adults: A tensor-based morphometry study. Journal of Alzheimer's Disease, 23(3), 433-442. doi: 10.3233/jad-2010-101398

Marstaller, L., Burianová, H., \& Reutens, D. C. (2016). Individual differences in structural and functional connectivity predict speed of emotion discrimination. Cortex, 85, 65-74. doi: 10.1016/j.cortex.2016.10.001

McIntosh, A. M., Moorhead, T. W. J., Job, D., Lymer, G. K. S., Maniega, S. M., McKirdy, J., ... Hall, J. (2007). The effects of a neuregulin 1 variant on white matter density and integrity. Molecular Psychiatry, 13, 1054-1059. doi: 10.1038/sj.mp.4002103 
Medland, S. E., Jahanshad, N., Neale, B. M., \& Thompson, P. M. (2014). Whole-genome analyses of whole-brain data: Working within an expanded search space. Nature Neuroscience, 17, 791-800. doi: 10.1038/nn.3718

Meyer-Lindenberg, A., Nicodemus, K. K., Egan, M. F., Callicott, J. H., Mattay, V., \& Weinberger, D. R. (2008). False positives in imaging genetics. Neurolmage, 40(2), 655-661. doi: 10.1016/j.neuroimage.2007.11.058

Mufford, M., Cheung, J., Jahanshad, N., van der Merwe, C., Ding, L., Groenewold, N., ... Psychiatric Genomics Consortium - Tourette Syndrome working group (2019). Concordance of genetic variation that increases risk for Tourette Syndrome and that influences its underlying neurocircuitry. Translational Psychiatry, 9, 120. doi: 10.1038/s41398-019-0452-3

Paus, T. (2005). Mapping brain maturation and cognitive development during adolescence. Trends in Cognitive Sciences, 9(2), 60-68. doi: 10.1016/j.tics.2004.12.008

Paus, T., Zijdenbos, A., Worsley, K., Collins, D. L., Blumenthal, J., Giedd, J. N., ... Evans, A. C. (1999). Structural maturation of neural pathways in children and adolescents: In vivo study. Science, 283(5409), 1908-1911. doi: 10.1126/science.283.5409.1908

Pennington, B. F., Filipek, P. A., Lefly, D., Chhabildas, N., Kennedy, D. N., Simon, J. H., ... DeFries, J. C. (2000). A twin MRI study of size variations in the human brain. Journal of Cognitive Neuroscience, 12(1), 223-232. doi: 10.1162/089892900561850

Peper, J. S., Zwiers, M. P., Boomsma, D. I., Kahn, R. S., \& Hulshoff Pol, H. E. (2009). Human brain volume: What's in the genes? In Kim, Y. K. (Ed.), Handbook of Behavior Genetics (pp. 137-157). New York: Springer. doi: 10.1007/978-0-387-76727-7 10

Pfefferbaum, A., Sullivan, E. V., Swan, G. E., \& Carmelli, D. (2000). Brain structure in men remains highly heritable in the seventh and eighth decades of life. Neurobiology of Aging, 21(1), 63-74. doi: 10.1016/s0197-4580(00)00086-5

Plomin, R., \& Craig, I. (1997). Human behavioural genetics of cognitive abilities and disabilities. BioEssays, 19(12), 1117-1124. doi: 10.1002/bies.950191211

Posthuma, D. (2002). Genetic variation and cognitive ability. Amsterdam: VU.

Posthuma, D., de Geus, E. J. C., Neale, M. C., Hulshoff Pol, H. E., Baaré, W. E. C., Kahn, R. S., \& Boomsma, D. (2000). Multivariate genetic analysis of brain structure in an extended twin design. Behavior Genetics, 30, 311-319. doi: 10.1023/A:1026501501434

Raz, N., Gunning-Dixon, F., Head, D., Rodrigue, K. M., Williamson, A., \& Acker, J. D. (2004). Aging, sexual dimorphism, and hemispheric asymmetry of the cerebral cortex: Replicability of regional differences in volume. Neurobiology of Aging, 25(3), 377-396. doi: 10.1016/s0197-4580(03)00118-0

Reveley, A. M., Reveley, M. A., Chitkara, B., \& Clifford, C. (1984). The genetic basis of cerebral ventricular volume. Psychiatry Research, 13(3), 261-266. doi: 10.1016/0165-1781(84)90041-6

Rimol, L. M., Agartz, I., Djurovic, S., Brown, A. A., Roddey, J. C., Kähler, A. K., ... Andreassen, O. A. (2010). Sex-dependent association of common variants of microcephaly genes with brain structure. Proceedings of the National Academy of Sciences, 107(1), 384-388. doi: 10.1073/pnas.0908454107

Scamvougeras, A., Kigar, D. L., Jones, D., Weinberger, D. R., \& Witelson, S. F. (2003). Size of the human corpus callosum is genetically determined: An MRI study in mono and dizygotic twins. Neuroscience Letters, 338(2), 91-94. doi: 10.1016/s0304394002013332

Schmitt, J. E., Lenroot, R. K., Wallace, G. L., Ordaz, S., Taylor, K. N., Kabani, N., ... Giedd, J. N. (2008). Identification of genetically mediated cortical networks: A multivariate study of pediatric twins and siblings. Cerebral Cortex, 18(8), 1737-1747. doi: 10.1093/cercor/bhm211 
ЗАХАРОВ И. М., МАЛЫХ С. Б.

ИССЛЕДОВАНИЯ СТРУКТУРНЫХ ХАРАКТЕРИСТИК МОЗГА В ПСИХОГЕНЕТИКЕ

РосСИЙскИй психологИЧЕСКИй жУРнАл, 2020, Т. 17, № 2, 17-35. doi: 10.21702/rpj.2020.2.2

МЕДИЦИНСКАЯ ПСИХОЛОГИЯ

Schmitt, J. E., Wallace, G. L., Lenroot, R. K., Ordaz, S. E., Greenstein, D., Clasen, L., ... Giedd, J. N. (2010). A twin study of intracerebral volumetric relationships. Behavior Genetics, 40, 114-124. doi: 10.1007/s10519-010-9332-6

Strike, L. T., Couvy-Duchesne, B., Hansell, N. K., Cuellar-Partida, G., Medland, S. E., \& Wright, M. J. (2015). Genetics and brain morphology. Neuropsychology Review, 25, 63-96. doi: 10.1007/s11065-015-9281-1

Thompson, P. M., Cannon, T. D., Narr, K. L., van Erp, T., Poutanen, V.-P., Huttunen, M., ... Toga, A. W. (2001). Genetic influences on brain structure. Nature Neuroscience, 4, 1253-1258. doi: $10.1038 / \mathrm{nn} 758$

Thompson, P. M., Jahanshad, N., Ching, C. R. K., Salminen, L. E., Thomopoulos, S. I., Bright, J., ... \& for the ENIGMA Consortium (2020). ENIGMA and global neuroscience: A decade of largescale studies of the brain in health and disease across more than 40 countries. Translational Psychiatry, 10, 100. doi: 10.1038/s41398-020-0705-1

Toga, A. W., \& Thompson, P. M. (2005). Genetics of brain structure and intelligence. Annual Review of Neuroscience, 28, 1-23. doi: 10.1146/annurev.neuro.28.061604.135655

van Haren, N. E. M., Hulshoff Pol, H. E., Schnack, H. G., Cahn, W., Brans, R., Carati, I., ... Kahn, R. S. (2008). Progressive brain volume loss in schizophrenia over the course of the illness: Evidence of maturational abnormalities in early adulthood. Biological Psychiatry, 63(1), 106-113. doi: 10.1016/j.biopsych.2007.01.004

van Leeuwen, M., van den Berg, S. M., Peper, J. S., Hulshoff Pol, H. E., \& Boomsma, D. I. (2009). Genetic covariance structure of reading, intelligence and memory in children. Behavior Genetics, 39, 245-254. doi: 10.1007/s10519-009-9264-1

van Soelen, I. L. C., Brouwer, R. M., Peper, J. S., van Beijsterveldt, T. C. E. M., van Leeuwen, M., de Vries, L. S., ... Boomsma, D. I. (2010). Effects of gestational age and birth weight on brain volumes in healthy 9 year-old children. The Journal of Pediatrics, 156(6), 896-901. doi: 10.1016/j. jpeds.2009.12.052

van Soelen, I. L. C., Brouwer, R. M., Peper, J. S., van Leeuwen, M., Koenis, M. M. G., van Beijsterveldt, T. C. E. M., ... \& Boomsma, D. I. (2012). Brain SCALE: Brain structure and cognition: An adolescent longitudinal twin study into the genetic etiology of individual differences. Twin Research and Human Genetics, 15(3), 453-467. doi: 10.1017/thg.2012.4

Vuoksimaa, E., Panizzon, M. S., Chen, C.-H., Fiecas, M., Eyler, L. T., Fennema-Notestine, C., ... \& Kremen, W. S. (2015). The genetic association between neocortical volume and general cognitive ability is driven by global surface area rather than thickness. Cerebral Cortex, 25(8), 2127-2137. doi: $10.1093 /$ cercor/bhu018

Wallace, G. L., Schmitt, J. E., Lenroot, R., Viding, E., Ordaz, S., Rosenthal, M. A., ... Giedd, J. N. (2006). A pediatric twin study of brain morphometry. Journal of Child Psychology and Psychiatry, 47(10), 987-993. doi: 10.1111/j.1469-7610.2006.01676.x

Walton, E., Hibar, D., Yilmaz, Z., Jahanshad, N., Cheung, J., Batury, V.-L., ... Ehrlich, S. (2019). Exploration of shared genetic architecture between subcortical brain volumes and anorexia nervosa. Molecular Neurobiology, 56, 5146-5156. doi: 10.1007/s12035-018-1439-4

Winkler, A. M., Kochunov, P., Blangero, J., Almasy, L., Zilles, K., Fox, P. T., ... Glahn, D. C. (2010). Cortical thickness or grey matter volume? The importance of selecting the phenotype for imaging genetics studies. Neurolmage, 53(3), 1135-1146. doi: 10.1016/j.neuroimage.2009.12.028 
Worsley, K. J., Marrett, S., Neelin, P., Vandal, A. C., Friston, K. J., \& Evans, A. C. (1996). A unified statistical approach for determining significant signals in images of cerebral activation. Human Brain Mapping, 4(1), 58-73. doi: 10.1002/(sici)1097-0193(1996)4:1<58::aid-hbm4>3.0.co;2-o

Wright, I. C., Sham, P., Murray, R. M., Weinberger, D. R., \& Bullmore, E. T. (2002). Genetic contributions to regional variability in human brain structure: Methods and preliminary results. Neurolmage, 17(1), 256-271. doi: 10.1006/nimg.2002.1163

Yoon, U., Fahim, C., Perusse, D., \& Evans, A. C. (2010). Lateralized genetic and environmental influences on human brain morphology of 8-year-old twins. Neurolmage, 53(3), 1117-1125. doi: 10.1016/j.neuroimage.2010.01.007

Yoon, U., Perusse, D., Lee, J.-M., \& Evans, A. C. (2011). Genetic and environmental influences on structural variability of the brain in pediatric twin: Deformation based morphometry. Neuroscience Letters, 493(1-2), 8-13. doi: 10.1016/j.neulet.2011.01.070

Zavala, C., Beam, C. R., Finch, B. K., Gatz, M., Johnson, W., Kremen, W. S., ... \& Reynolds, C. A. (2018). Attained SES as a moderator of adult cognitive performance: Testing gene-environment interaction in various cognitive domains. Developmental Psychology, 54(12), 2356-2370. doi: $10.1037 /$ dev0000576

Zuliani, R., Moorhead, T. W. J., Bastin, M. E., Johnstone, E. C., Lawrie, S. M., Brambilla, P., ... Mclntosh, A. M. (2011). Genetic variants in the ErbB4 gene are associated with white matter integrity. Psychiatry Research: Neuroimaging, 191(2), 133-137. doi: 10.1016/j.pscychresns.2010.11.001

\section{Конфликт интересов отсутствует}

